\title{
Design of a portable imager for near- infrared visualization of cutaneous wounds
}

Zhaoqiang Peng

Jun Zhou

Ashley Dacy

Deyin Zhao

Vasant Kearney

Weidong Zhou

Liping Tang

Wenjing $\mathrm{Hu}$ 


\title{
Design of a portable imager for near-infrared visualization of cutaneous wounds
}

\author{
Zhaoqiang Peng, ${ }^{\mathrm{a}, \dagger}$ Jun Zhou, ${ }^{\mathrm{b}, \dagger}$ Ashley Dacy, ${ }^{\mathrm{b}}$ Deyin Zhao, ${ }^{\mathrm{a}}$ Vasant Kearney, ${ }^{\mathrm{b}}$ Weidong Zhou, ${ }^{\mathrm{a}}$ \\ Liping Tang, ${ }^{b, *}$ and Wenjing $\mathrm{Hu}^{\mathrm{c}}$ \\ aUniversity of Texas at Arlington, Department of Electrical Engineering, P.O. Box 19016, Arlington, Texas 76019, United States \\ bUniversity of Texas at Arlington, Department of Bioengineering, P.O. Box 19138, Arlington, Texas 76019, United States \\ 'Progenitec Inc., 917 Parktree Drive, Arlington, Texas 76015, United States
}

\begin{abstract}
A portable imager developed for real-time imaging of cutaneous wounds in research settings is described. The imager consists of a high-resolution near-infrared CCD camera capable of detecting both bioluminescence and fluorescence illuminated by an LED ring with a rotatable filter wheel. All external components are integrated into a compact camera attachment. The device is demonstrated to have competitive performance with a commercial animal imaging enclosure box setup in beam uniformity and sensitivity. Specifically, the device was used to visualize the bioluminescence associated with increased reactive oxygen species activity during the wound healing process in a cutaneous wound inflammation model. In addition, this device was employed to observe the fluorescence associated with the activity of matrix metalloproteinases in a mouse lipopolysaccharide-induced infection model. Our results support the use of the portable imager design as a noninvasive and real-time imaging tool to assess the extent of wound inflammation and infection. $\odot 2017$ Society of PhotoOptical Instrumentation Engineers (SPIE) [DOI: 10.1117/1.JBO.22.1.016010]
\end{abstract}

Keywords: imaging device; optical imaging; wound healing; diagnosis.

Paper 160472RR received Jul. 6, 2016; accepted for publication Dec. 27, 2016; published online Jan. $13,2017$.

\section{Introduction}

The management of skin wounds is a pervasive issue in modern medicine. Chronic wounds affect over 6.5 million patients and cost more than $\$ 50$ billion per year in health care costs in the United States alone. With an increasing senior citizen population and incidence of diabetes and obesity worldwide, it is anticipated that human skin wound care will become a snowballing threat to the economy and public health. ${ }^{1}$ To overcome this challenge, substantial research efforts have been focused on the development of new treatments for chronic wounds, including extracellular matrix scaffolds, negative pressure wound therapy, and hyperbaric oxygenation. ${ }^{2}$ However, little progress has been made on the diagnosis and monitoring of the progress of chronic wound healing. Over the past three decades, several noninvasive methods have been developed to provide an objective assessment of the wound healing process, including high-resolution ultrasound, ${ }^{3}$ confocal microscopy, ${ }^{4}$ thermography, ${ }^{5}$ laser Doppler imaging, ${ }^{6}$ polarization imaging, and optical coherence tomography. ${ }^{7,8}$ These techniques provide reasonable structural resolution and, most importantly, allow visualization of newly formed tissue underneath a scab. However, these techniques primarily focus on the observation of re-epithelialization, revascularization, reformation of the dermal-epidermal junction, and events that relate mainly to late phases of the healing process. These diagnostic methods also do not provide the capability to identify specific biochemical signals that reflect the extent of wound healing and tissue regeneration. Therefore, there is an urgent need for the development of new technology to monitor chronic wounds.

\footnotetext{
*Address all correspondence to: Liping Tang, E-mail: Itang@uta.edu
}

${ }^{\dagger}$ Both authors contributed equally to this work.
Near-infrared (NIR) and luminescence optical imaging have become promising approaches for noninvasive tissue characterization. NIR light between 750 and $900 \mathrm{~nm}$ is minimally absorbed by tissue components that typically interfere with in vivo imaging, such as melanin and hemoglobin, and preferentially scattered, allowing deep tissue propagation. ${ }^{9,10}$ NIR imaging is sensitive to the volume of tissue that falls between the source light entering the tissue and the detector, receiving light that diffuses out of the tissue. This imaging technology has the ability to differentiate diseased tissues from healthy tissues at a molecular level, as well as the potential to provide complementary information to current clinical imaging techniques without harmful radiation, radioactive substances, or ungainly instrumentation. ${ }^{11}$ NIR imaging systems have been used to image oxygenation to visualize the healing status of the wound bed in diabetic ulcers. ${ }^{12}$ Optical contrast has also been used to analyze diabetic foot ulcers. ${ }^{13}$ Equally important, imaging probes have been developed to provide real-time monitoring of the extent of several wound healing markers and byproducts, including matrix metalloproteinases (MMPs), $\mathrm{pH}$, and reactive oxygen species (ROS).${ }^{14}$ Some NIR imaging probes have also been developed to detect the accumulation and activation of neutrophils and macrophages at cutaneous wounds sites. ${ }^{15,16}$ In addition, an imaging method has been developed to detect bacterial infections in wounds. ${ }^{17}$ Additionally, luminescence imaging in wound healing has been applied to utilize the native bioluminescence of $E$. coli to quantify wound infection. ${ }^{18}$ Several probes have also been developed that luminesce upon contact with markers of wound healing, such as changing $\mathrm{pH}$ and ROS production. ${ }^{19,20}$ For example, L012, a luminescent ROS probe, has been utilized to accurately detect skin 
inflammatory responses and infection. ${ }^{19}$ Many of these probes are attractive targets for development into platform technologies for wound monitoring.

To translate imaging probe technology into clinical diagnosis, there is a need for portable imaging systems that can detect both luminescent and fluorescent signals on wounds. Although several optical imagers with this capacity are commercially available, ${ }^{19}$ such devices are designed as large enclosed units for small animal research and cannot be used for large animals or human subjects. Several portable imager prototypes have been developed with the capability to visualize wounds. Several targets have been utilized. ${ }^{21}$ For example, skin wounds in diabetic rats have been characterized using a simple fNIR (functional NIR spectroscopic) device consisting of a Teflon laser probe with one source and four detector fibers and diffuse optical tomography. ${ }^{22,23}$ However, the relatively poor resolution that results from the high degree of light scatter in biological tissue limits their application. ${ }^{24}$ Recently, an inexpensive camera with a polarizing filter was tested for the determination of wound area in $2010 .{ }^{25}$ In 2011, an imaging device capable of measuring partial pressure of oxygen and $\mathrm{pH}$ in a two-dimensional (2-D) image was fabricated using a digital single-lens reflex camera and a $405-n m$ LED light source. ${ }^{26}$ While these devices are portable and easy to use, they lack the ability to visualize fluorescent signals due to the lack of proper light sources and emission filters. Therefore, there remains a need for lowcost portable imaging devices to visualize wide ranges of fluorescent and luminescent signals with high sensitivity and resolution.

Here we report the development of a portable imaging system that incorporates NIR fluorescence and luminescence imaging modalities for real-time imaging. The portable imaging system is composed of a CCD camera, LED ring light source, and emission filters similar to many commercial systems. ${ }^{20,26}$ To optimize portability, this device integrates all components into a small "black box" setup with easily interchangeable LED rings for different excitation wavelengths, eliminating the need for excitation filters. The LED ring makes the system compact without sacrificing performance. A rotatable filter wheel allows the selection of different emission wavelengths with ease for varying situations. The device is compact and portable, allowing easy transportation to different sites in a research setting. All necessary components are integrated into an easy-to-use external camera attachment. The entire system can be electronically controlled except the filter wheel, which has a manually controllable section outside the box. The versatility of this mount-andplay device permits its use for animal research and also displays potential for human wound diagnosis.

\section{Materials and Methods}

\subsection{Materials}

An ORCA-R2 CCD camera (C10600) was obtained from Hamamatsu. A 5-mm focal length $f / 1.4$ lens (MVL5M23), an LED driver (DCZ100), and a 60-mm cage system filter wheel (LCFW5) were obtained from Thorlabs. Two-inch-diameter thin-film $700 \pm 2 \mathrm{~nm}$ and $810 \pm 2 \mathrm{~nm}$ NIR filters (67-905, 67-916) were obtained from Edmund Optics. A $630 \pm 10 \mathrm{~nm}$ and a $740 \pm 10 \mathrm{~nm}$ LED ring light were obtained from ProPhotonix (RF2-630-VXF100). The portable imager housing was constructed using in-house laser-cut 5-mm-thickness black polycarbonate.

\subsection{Device Design Development}

The portable imager was designed to be compact and portable to enable monitoring of the wound healing process using various fluorescence and luminescent imaging probes. The specifications for the imaging system are summarized and compared with a commercial animal imager (Kodak In Vivo FX Pro imaging system, Kodak) in Table 1.

The imager has four major components, including a CCD camera, filter wheel, LED rings, computer, and LED driver [Fig. 1(a)]. The portable imager setup and a schematic of its light path are shown in Fig. 1(b).

The design incorporates a single-wavelength LED ring as the light source. The LED ring is interchangeable based on the required excitation wavelength, providing uniform excitation light onto the samples below. The intensity of the excitation light can be controlled via driver software provided by the manufacturer.

The emitted NIR light passes through the center opening of the LED ring and is collected by the CCD camera through a bandpass filter. A manually controlled filter wheel allows the user to easily change the emission wavelength. The complete optical imaging system is mounted inside a black polycarbonate casing designed to efficiently block ambient light. White light images were generated by setting the filter wheel to the open position with excitation by ambient room lighting, which was found to produce clear, high-resolution images. In addition, the LED ring can remain off and the filter wheel set to the open position to allow the imager to detect bioluminescence.

Although the comparison of the portable imager design with the Kodak system was as direct as possible, it was inherently

Table 1 Comparison of the specifications of the portable imager and a Kodak In Vivo FX Pro imaging system.

\begin{tabular}{|c|c|c|c|}
\hline & Specification & Portable imager & $\begin{array}{c}\text { Kodak In Vivo } \\
\text { FX Pro }\end{array}$ \\
\hline \multirow[t]{4}{*}{ Detector } & Pixel size & $5-10 \mu \mathrm{m}$ & $7.4 \mu \mathrm{m}$ \\
\hline & Dynamic range & 16 bit & 16 bit \\
\hline & Dark current & $<0.001 \mathrm{e} / \mathrm{pixel} / \mathrm{s}$ & $0.003 \mathrm{e} / \mathrm{pixel} / \mathrm{s}$ \\
\hline & Read noise & $\begin{array}{c}<100 \text { electrons } \\
\text { (rms) }\end{array}$ & $<9$ electrons (rms) \\
\hline \multirow[t]{3}{*}{ Light source } & Type & LED & Xenon bulb \\
\hline & Wavelengths & 630 or $740 \mathrm{~nm}$ & $\begin{array}{l}410-760 \mathrm{~nm} \\
\text { (excitation) }\end{array}$ \\
\hline & & & $\begin{array}{c}\text { 535-830 nm } \\
\text { (emission) }\end{array}$ \\
\hline \multirow[t]{2}{*}{ Filter } & Type & Thin-film coatings & Thin-film coatings \\
\hline & Transmission & $>60 \%$ & $>60 \%$ \\
\hline Zoom lens & Angle of view & $>28 \mathrm{deg}$ & $33 \mathrm{deg}$ \\
\hline \multirow[t]{2}{*}{ Dimensions } & $\begin{array}{c}\text { Size } \\
(L \times W \times H)\end{array}$ & $22 \times 22 \times 25 \mathrm{~cm}$ & $104 \times 61 \times 96.5 \mathrm{~cm}$ \\
\hline & Weight & $5.8 \mathrm{~kg}$ & $142 \mathrm{~kg}$ \\
\hline
\end{tabular}


limited. While the height of the portable imager can be adjusted to change the distance between the camera and objective, the camera of Kodak Imager is contained in a sealed box and its distance cannot be adjusted. Additionally, the excitation light intensity of the portable imager's LED ring can be adjusted while the intensity of the Kodak imager's bulb is fixed. Image acquisition was also controlled with different manufacturer-provided software for each machine: Carestream ${ }^{\circledR}$ software for the Kodak imager and HCImage Live ${ }^{\circledR}$ for the portable imager. Despite these differences, useful comparisons were able to be drawn between these two imagers when contrasting their detection sensitivities of fluorescent/bioluminescent signals in vitro and in vivo. To ensure this, all images were taken with the same excitation $(630 \mathrm{~nm})$ and emission $(700 \mathrm{~nm})$ wavelengths and exposure time. To eliminate the potential variation due to image analysis software, images from both machines were analyzed using WoundView ${ }^{\circledR}$ software in an identical manner.

\subsection{Image Processing}

An in-house image processing software platform (WoundView $^{\circledR}$ ) allows the user to generate manual, semiautomatic, and fully automatic regions of interest (ROIs). WoundView ${ }^{\circledR}$ generates statistical information from the ROIs and allows the user to superimpose up to three images at a time. For studies involving multiple time points, this allows images to be overlaid and ROIs to be automatically extrapolated to generate ROIs over the same area measured in the initial image. The manual ROI generation tool allows the user to draw freehand or use an adjustable ellipse. The semiautomatic tool allows the user to adjust an intensity threshold within the ROI. The fully automatic tool allows the user to select a contiguous area of interest from which the algorithm will generate an ROI from a userspecified isointensity level. WoundView ${ }^{\circledR}$ utilizes a MATLAB ${ }^{\circledR}$ (Mathworks, Inc.) based interface with object-oriented $\mathrm{C}++$ wrapped functions (Microsoft Visual Studios) for computational efficiency. Figure 2 shows a representative background image with a superimposed intensity image with four semiautomatically generated ROIs. Isointensity lines are displayed ranging from blue (10\% maximum intensity) to red (90\% maximum intensity).

ROIs for the areas of interest were manually selected using the freeform drawing tool. The same threshold (70\%) was

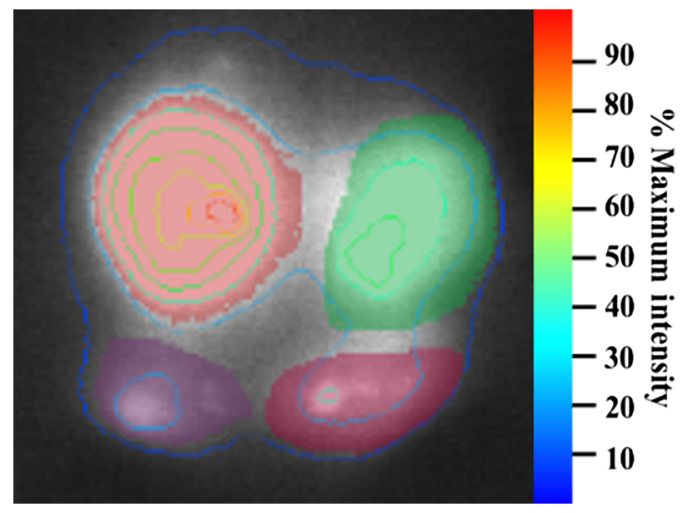

Fig. 2 Generation of four different semiautomatic ROls superimposed on a white light background image. Isointensity lines are displayed ranging from blue (10\% maximum intensity) to red $(90 \%$ maximum intensity).

applied for each ROI. Average intensities were calculated for each ROI using WoundView ${ }^{\circledR}$ software. Three ROIs were measured to calculate averages and standard deviations.

\subsection{Device Characterization}

The portable imager's single-wavelength LED ring excitation design was characterized by comparing with a commercial product (Kodak In Vivo FX Pro) using identical samples, experimental setup, and camera settings. To compare the beam uniformity among all systems, the optimal working distance was determined by taking beam uniformity measurements at different distances. For in vitro fluorescence measurements, all measurements used a 1.5-mL Eppendorf tube filled with 1-mL Cy5 (Lumiprobe Co.), an NIR dye. In vivo sensitivity measurements for both fluorescence and luminescence were performed in a murine subcutaneous dye injection model.

\subsection{In Vivo Imaging in Mouse Models}

Balb/c mice (female, aged three months) were used in all in vivo studies. Two well-established mouse models-an excisional wound model and an acute inflammation model-were used to evaluate the capability of the portable imager to detect various (a)

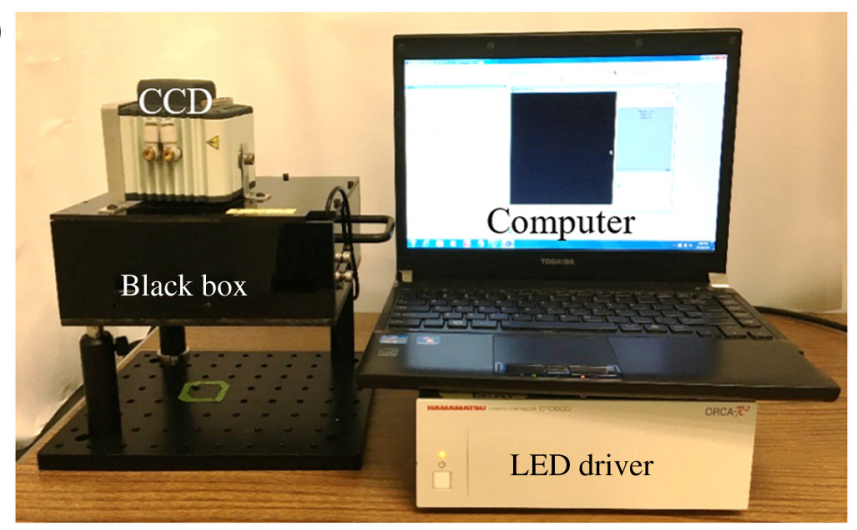

(b)

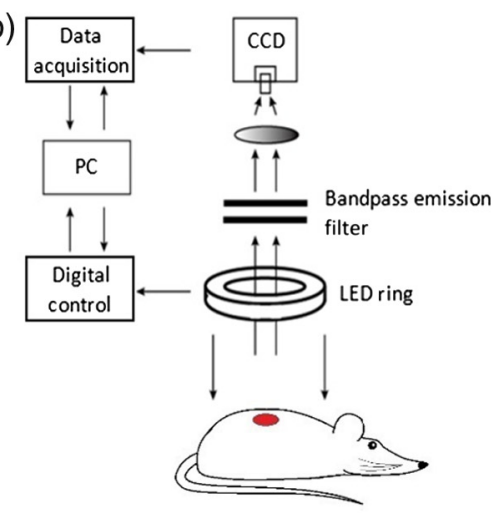

Fig. 1 Setup and light schematic of the main components of the portable imager. (a) Major components of portable imager, including a CCD camera, the black box (containing filter wheel and LED ring), a computer running WoundView ${ }^{\circledR}$ software, and an LED driver. (b) Schematic and light path of the portable imager design. 
levels of inflammatory reaction and infection in vivo. All animal protocols were approved by the Animal Studies Committee at the University of Texas at Arlington.

An excisional wound model was created to simulate wound healing responses. ${ }^{27}$ It is well established that ROS play an important role in wound healing processes. ${ }^{28}$ In addition, an ROS-sensitive luminescent dye, L-012, has been shown to emit luminescent signals in response to ROS activities in vivo. ${ }^{19}$ Briefly, 1-day- and 6-day-old skin incision wounds ( $2 \mathrm{~mm}$ diameter, 2 wounds/animal) were created on the back of 3 three-month-old female BALB/c mice. Immediately before imaging, wounds and surrounding healthy skin (as control) were treated topically with $10 \mu \mathrm{L}$ of $52 \mathrm{mM} \mathrm{L-012}$ (Wako Chemicals, Inc. Richmond, Virginia). Luminescent signals in and surrounding the wounds were then imaged for $20 \mathrm{~min}$. Wound-associated luminescent signals were quantified using WoundView ${ }^{\circledR}$ and then statistically analyzed. Animals were anesthetized with $2 \%$ isoflurane during all procedures.

A subcutaneous infection model was employed to investigate the dynamics of MMP activity following the subcutaneous injection of the bacterial toxin lipopolysaccharide (LPS). ${ }^{19,29}$ Briefly, $20 \mu \mathrm{L}$ of LPS $(3.61 \mathrm{mM})$ or PBS buffer $(50 \mathrm{mM}$, control) mixed with $100 \mu \mathrm{L}$ of MMP-sensitive fluorescent probe ( $2 \mathrm{nM}$, MMPSense750 FAST, PerkinElmer, Waltham, Massachusetts) were injected into the subcutaneous cavity. Thirty minutes after injection, fluorescent images of the animals were taken with a 740-nm LED ring and 810-nm emission filter with an exposure time of $20 \mathrm{~s}$. The fluorescent signals in and surrounding the wounds were quantified using WoundView ${ }^{\circledR}$ software. Animals were anesthetized with $2 \%$ isoflurane during all procedures.

\subsection{Statistical Analyses}

Statistical analysis between different treatment groups was carried out using a Student's $t$-test. Differences were considered statistically significant when $P \leq 0.05$. Linear regression analyses were used to determine the relationship between dye concentrations and fluorescent intensities and ROS activities and luminescent intensities. The coefficient of determination $\left(R^{2}\right)$ was calculated to provide a measure of correlation.

\section{Results}

\subsection{Device Characterization}

Beam uniformity of a 630-nm LED ring was compared with the Kodak machine's 175 W xenon bulb equipped with a 630-nm excitation filter. White paper was used as a test medium for the light beams. To compare the two systems, experimental conditions were kept consistent with the same maximum light intensity of $22.5 \mu \mathrm{W} / \mathrm{cm}^{2}$ (determined using a thermal power meter). Results are presented in Fig. 3 for the LED ring [Fig. 3(a)] and xenon bulb [Fig. 3(b)] as normalized intensity, calculated as light intensity at a given point divided by maximum intensity over the illumination area.

Beam uniformity can be determined mathematically in a oneand two-dimensional case by the uniformity index (UI). ${ }^{30}$ Here, we used a 2-D UI to describe and compare these two systems. For $f(x, y)$ representing the intensity distribution of the light field on $x$ and $y$ axes, UI can be defined as (a)
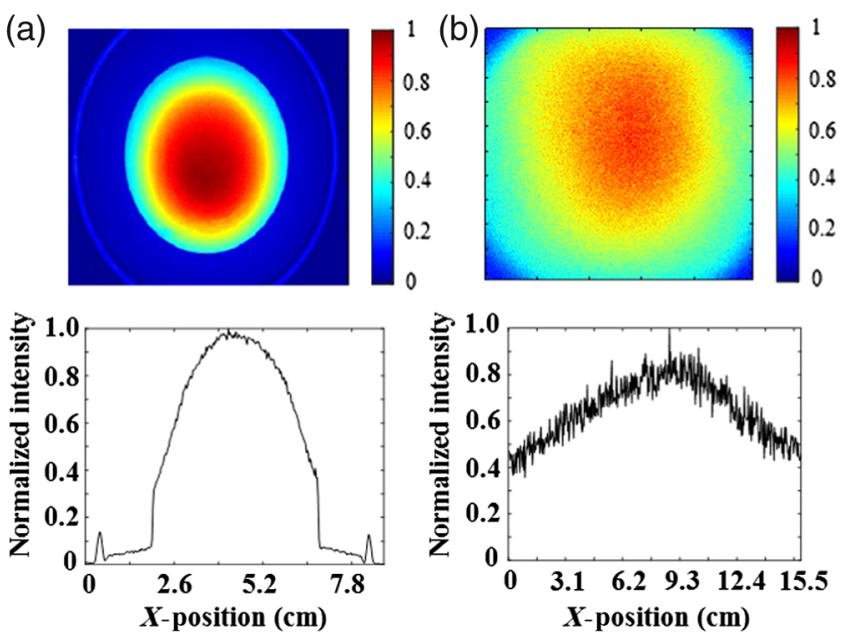

Fig. 3 Beam uniformity was compared between the LED ring of (a) the portable imager and (b) xenon blub of the Kodak in vivo imager. The beam intensities were normalized based on the maximum intensity in the center of the illumination area. Normalized beam intensity is reported (top) with graphical representations of the data (bottom). The xenon blub of the Kodak imager displayed good uniformity, while the LED ring of portable imager showed a good combination of high beam uniformity and low noise.

$\mathrm{UI}=\frac{\int \sqrt{\left(\frac{\mathrm{d} f}{\mathrm{~d} x}\right)^{2}+\left(\frac{\mathrm{d} f}{\mathrm{~d} y}\right)^{2}} \mathrm{~d} x \mathrm{~d} y}{\int f(x, y) \mathrm{d} x \mathrm{~d} y}\left(\mathrm{~m}^{-1}\right)$.

In this equation, if the derivative terms in the numerator are larger than the integral term in the denominator, a larger index will result. Conversely, better light homogeneity results in a smaller index. The overall UI of the Kodak imager is $0.0686 \mathrm{~m}^{-1}$ compared to $0.0361 \mathrm{~m}^{-1}$ for the portable imager's LED ring. Less fluctuation in the lighting intensity gives rise to more uniform image area. The view limitation and light convergence also contribute to the LED ring's UI. After shrinking integral range to effective working area $[2.8 \mathrm{~cm}(W) \times 3.5 \mathrm{~cm}(L)]$, the UI of the LED ring is $0.0122 \mathrm{~m}^{-1}$. A smaller uniform area was observed for the portable imager's LED ring compared to the Kodak's light source. However, with its portability and lack of area constraints, large wounds can still be analyzed by using processing software to combine several real-time images. Positioning the open device to take several images of large chronic wounds provides good flexibility to compensate for a smaller uniform area.

The LED ring has a divergence angle that alters uniformity with distance, making it necessary to calibrate the optimal working distance. Working distance was defined as the length from the sample surface to bottom surface of the black box. This value has the capacity to be easily adjusted for various applications.

The manufacturer's recommended working distance of the LED ring is $4 \mathrm{~cm}$. After calibration (Fig. 4 ), 4 to $5 \mathrm{~cm}$ was determined to give the most uniform light. The field of view is $8.8 \mathrm{~cm}(W) \times 8.8 \mathrm{~cm}(L)$, and the uniform area, defined as the illumination area with light intensity above $80 \%$ of the maximum, is $2.8 \mathrm{~cm}(W) \times 3.5 \mathrm{~cm}(L)$.

For a working distance of $3 \mathrm{~cm}$, the intensity of light at the center is much weaker than the edge, which means the lights from each LED do not overlap in the center. At 4, 5, and 

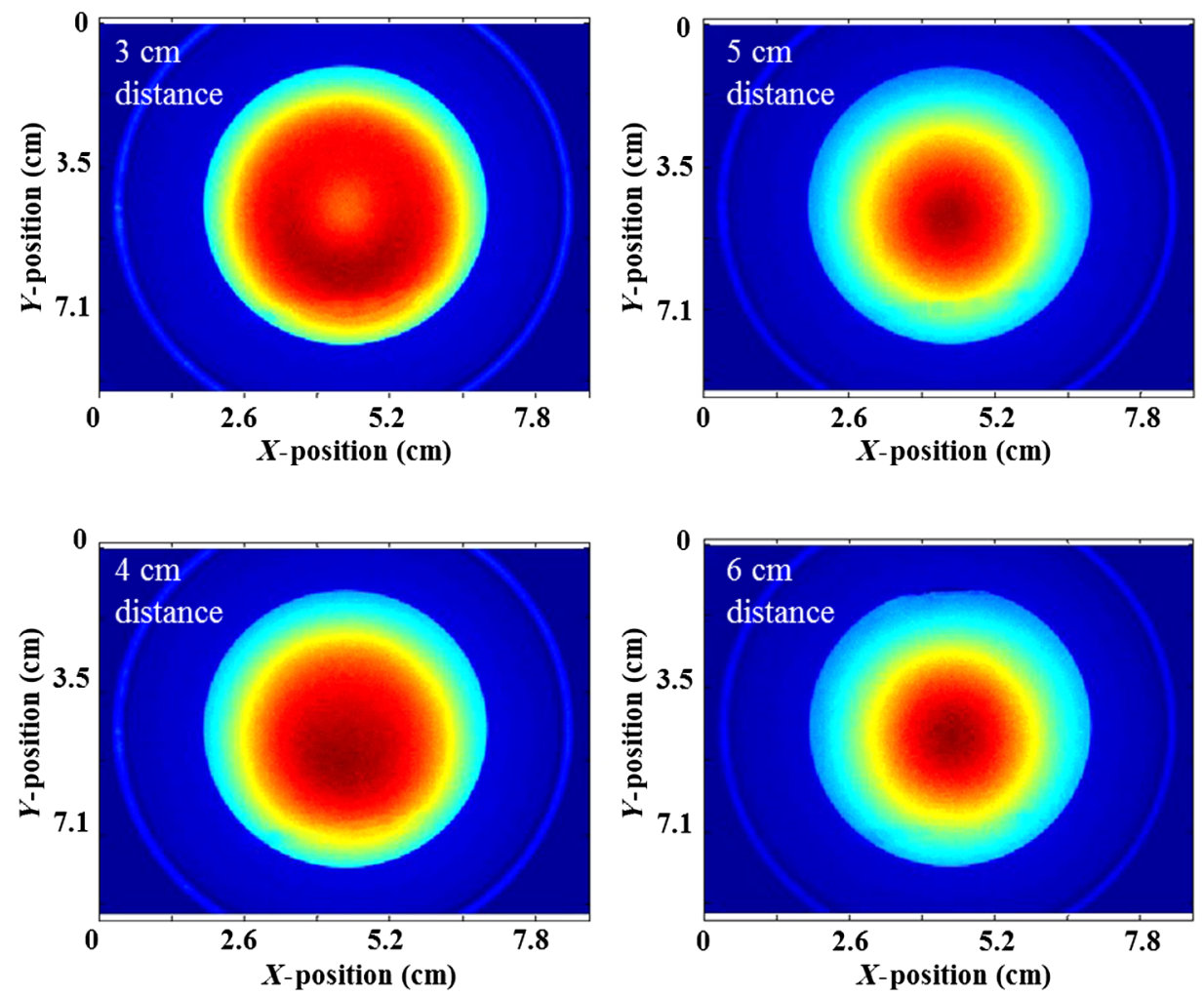

Fig. 4 Working distance of the portable imager was calibrated by comparing beam uniformity at different distances $(3,4,5$, and $6 \mathrm{~cm})$. Optimal beam uniformity was obtained at $4 \mathrm{~cm}$.
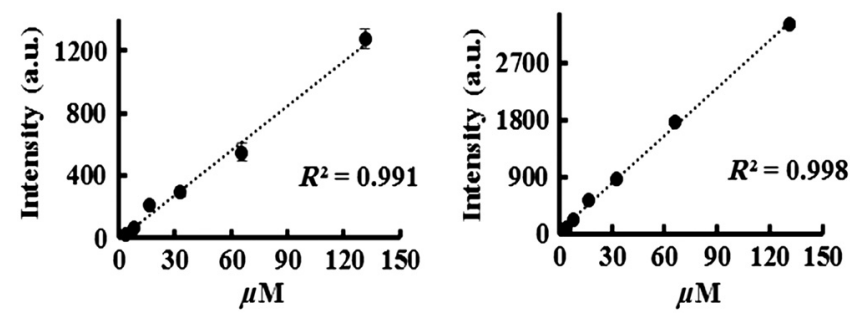

Fig. 5 Several concentrations of Cy5 dye were correlated with fluorescence intensities using an excitation wavelength of $630 \mathrm{~nm}$ with both (a) the portable imager and (b) the Kodak system.
$6 \mathrm{~cm}$, the intensity of light at the center is a stronger than at the edge, where it decreases with distance. Based on the homogeneity of the light intensity, $4 \mathrm{~cm}$ was chosen as the optimal working distance.

The relationship between dye concentration and fluorescent intensity was compared between the portable imager and the Kodak imaging system. Six concentrations of Cy5 dye (131.26, 65.6, 32.8, 16.4, 8.2, and $4.1 \mu \mathrm{M})$ were excited with $630 \mathrm{~nm}$ light. The fluorescent intensities from both imagers were quantified and statistically analyzed. In general, intensity values for the portable imager were slightly lower than the Kodak system (Fig. 5). However, both systems showed an excellent linear relationship between dye concentrations and fluorescent intensity $\left(R^{2}=0.991\right.$ and 0.998 for portable imager and Kodak imaging system, respectively). These results support that both imagers are capable of measuring and quantifying fluorescent intensities in vitro. It was worth noting that both imaging (a)

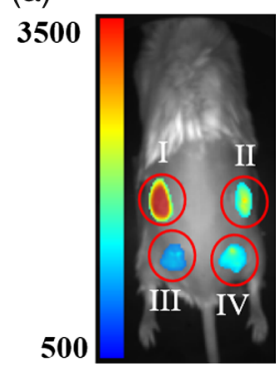

(b)

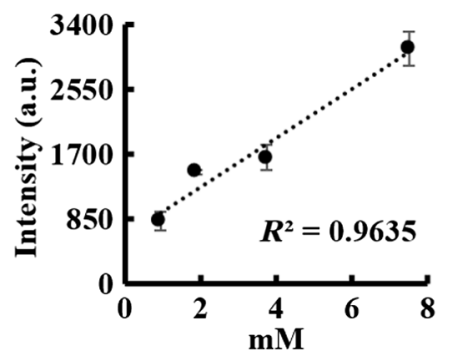

(c)

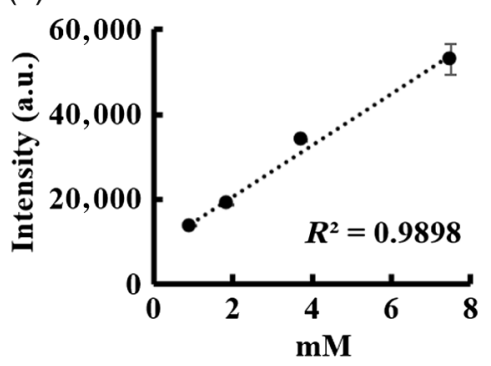

Fig. 6 In vivo fluorescent imaging of Cy5 dye in mice. (a) Representative fluorescent image of Cy5 dye in different concentrations [(I) 7.5, (II) 3.75 , (III) 1.88 , and (IV) $0.94 \mathrm{mM}$ ] using the portable imager. Linear relationships between dye concentrations and fluorescent intensities were determined based on imaging results obtained from (b) the portable imager and (c) the Kodak system. 
(a)

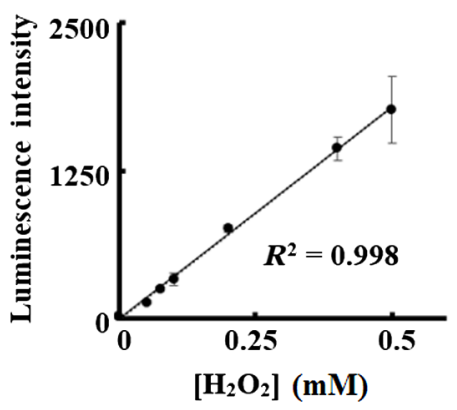

(b)

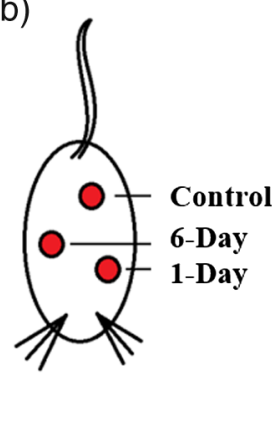

(c)

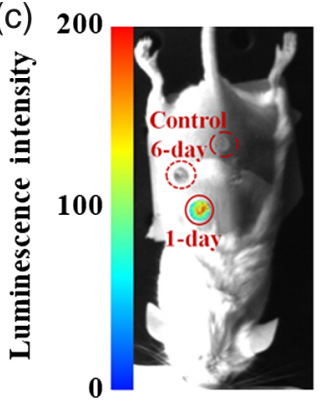

(d)

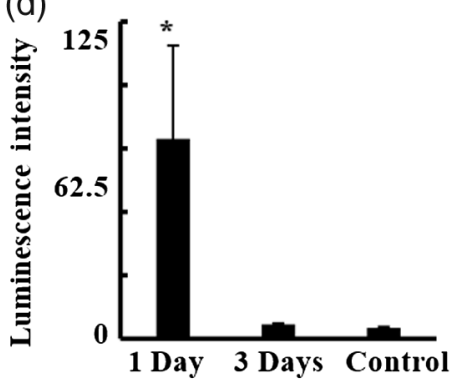

Fig. 7 Luminescent imaging of ROS activities in vitro and in vivo. (a) A linear relationship was observed between increasing ROS-producing $\mathrm{H}_{2} \mathrm{O}_{2}$ and luminescent intensity in vitro. (b) An illustration of an excisional wound mouse model with different age wounds (1-day and 6day) and healthy skin as control. (c) A representative luminescent image shows wounds of different ages display varying degrees of ROS activity. (d) The ROS activities in 1-day and 6-day wounds and controls were quantified and statistically analyzed.

systems were unable to detect signals from Cy5 dye clearly below dye concentrations of $4.1 \mu \mathrm{M}$, indicating that the portable imaging system has detection limits similar to the Kodak imaging system.

The in vivo sensitivity of both imagers was tested using a murine subcutaneous injection model. Four concentrations of Cy5 dye $(7.50,3.75,1.88$, and $0.94 \mathrm{mM})$ were injected under the skin in discrete locations on the dorsal area of deceased mice. Images were then taken immediately using both the Kodak and portable imagers and the fluorescence intensities were quantified [Fig. 6(a)]. It was determined that both systems had good linear relationships between dye concentrations and fluorescence intensities [Figs. 6(b) and 6(c)].

\subsection{In Vivo Imaging of Skin Inflammation in Mice}

The capability of the portable imager to detect luminescent signals was evaluated using both in vitro and in vivo models. The ROS-reactive luminescent probe L-012 (Wako) was utilized, having been shown to possess excellent sensitivity for quantification of ROS activities during inflammatory responses both in vitro and in vivo. ${ }^{19}$

In vitro tests were carried out to determine if the portable imager could detect ROS. Specifically, $\mathrm{H}_{2} \mathrm{O}_{2}$ was selected as an ROS-producing agent following previous literature. ${ }^{19}$ Twenty- $\mu \mathrm{L}$ of L-012 (48 mM) was mixed with $200 \mu \mathrm{L}$ of $\mathrm{H}_{2} \mathrm{O}_{2}$ in aqueous solution ( 0 to $0.5 \mathrm{mM}$ ) in the wells of a 96-well plate. Luminescence intensities were recorded using the portable imager with an exposure time of $20 \mathrm{~min}$ [Fig. 7(a)]. An increase in luminescence intensities was observed with increasing $\mathrm{H}_{2} \mathrm{O}_{2}$ concentrations. Statistical analyses showed a linear relationship between luminescent intensities and $\mathrm{H}_{2} \mathrm{O}_{2}$ concentrations, suggesting that the portable imager can detect $\mathrm{H}_{2} \mathrm{O}_{2}$-associated luminescent signals and quantify $\mathrm{H}_{2} \mathrm{O}_{2}$ concentration in vitro.

In vivo imaging was conducted using a mouse excisional wound model to evaluate the ability of the portable imager to detect ROS-associated luminescent signals in skin wounds. As shown in Figs. 7(b) and 7(c), the portable imager was able to measure ROS in wounds at different stages [Figs. 7(c) and 7(d)]. Significantly stronger ROS signal was observed in 1-day wounds, which are known to have inflammatory cell responses and maximal ROS activities [Fig. 7(c)]. ROS activities were significantly lower in 6-day wounds (at the end of acute inflammatory processes) and in control skin than in 1-day wounds [Fig. 7(d)]. These observations are in good agreement with previous findings. ${ }^{31}$

Previous studies have demonstrated that MMPs participate in infectious diseases' inflammatory responses and can be upregulated in response to the presence of bacterial toxins. ${ }^{32-34}$ Using a subcutaneous mice skin infection model [as shown in Fig. 8(a)] and commercially available MMP-sensitive fluorescent probe (MMPSense750, PerkinElmer, Inc.), the ability of the portable imager to measure the production and release of MMPs was determined. As expected, the portable imager was found to easily detect the production of MMPs [Fig. 8(b)]. A significant increase in fluorescent signal was observed at the LPS treatment site compared to a PBS control. Image analysis showed (a)

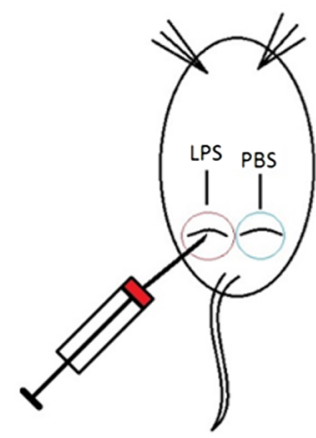

(b)

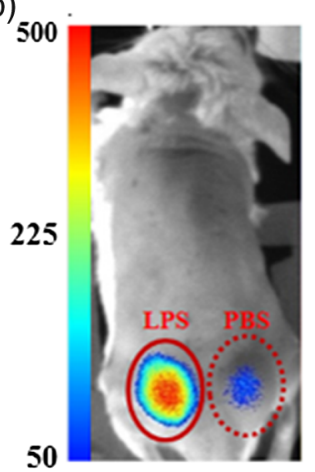

(c)

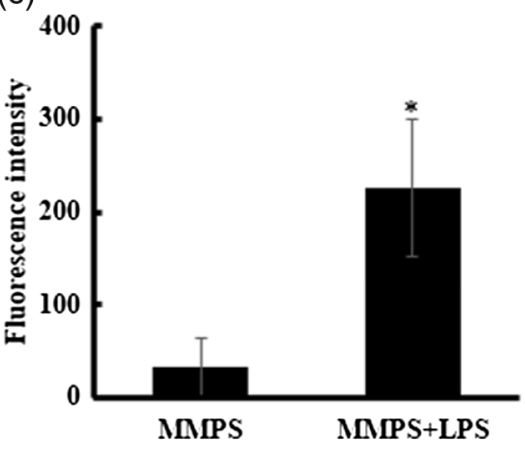

Fig. 8 Quantification of MMP activity in vivo using the portable imager. (a) An illustration of a skin infection model with subcutaneous injection of LPS and PBS (control). (b) Representative fluorescent image displaying different extents of fluorescent signals emitted by MMP-sensitive fluorescent probe injected subcutaneously with or without the presence of LPS. (c) Quantification of fluorescent intensities demonstrated that LPS treatment significantly increased MMP activity in vivo. 
approximately eight times higher fluorescent intensity in the LPS treatment group than PBS treatment [Fig. 8(c)]. These results are in agreement with previous studies. ${ }^{32-34}$

\section{Discussion and Conclusions}

Many NIR and luminescent probes have been developed to detect inflammatory response and infection in wounds. ${ }^{16,17,19,20}$ However, these exciting research advancements only benefit animal studies for which commercial imagers are available for the detection of fluorescent and luminescent signals in small animals. All commercial imagers share the same enclosed black box design and cannot be converted for disease diagnosis on large animals or patients. To overcome this limitation, an open and portable imager was developed. To assess its sensitivity and accuracy, the portable imager was evaluated and compared to a commercial small animal imaging device (Kodak In Vivo Imaging System). It is well established that this system, like many other commercial products, has high detection sensitivity and accuracy.

The portable imager design uses an LED ring/CCD setup, which eliminates the need for total imaging area enclosure and excitation filters, allowing the system to image an open surface without problems with ambient light. The imager has a compact and flexible design, removing the need for bulky equipment that restricts the usage due to subject size and geometry. The entire system can easily be transported to allow imaging of a subject in the most convenient location. It is also inexpensive compared to conventional in vivo wound imaging methods. The portable imager is condensed into an external camera attachment and utilizes MATLAB ${ }^{\circledR}$-based software that is reliably user-friendly and gives the user extensive options for image analysis.

When compared with a commercially available in vivo imaging system from Kodak, the proposed design stands toe-totoe. The LED ring produces an impressively uniform, symmetrical beam upon exposure to a sample. Although the Kodak imager produced a wider Gaussian peak, it displayed more noise over the uniform area. The imager was able to detect very low concentrations of dye despite inherent limitations from reduced biosensors and quantum efficiency, proving its feasibility for use in in vivo imaging applications. In short, although the Kodak system was shown to have better sensitivity and a larger view area, our device has significant potential to improve upon current imaging technology with further improvements, which will include developing a custom light source for a wider uniform illumination area.

The portable imager design was shown to capably monitor wound environment and conditions using several biomarkers and commercially available luminescent and fluorescent probes. In a wound inflammation model, ${ }^{35,36}$ the new device was able to measure luminescent signals emitted from an ROS-detecting probe in acute wounds, providing proof-of-concept for the suitability of this device for wound visualization applications. In addition, by quantifying fluorescent signals emitted from MMP-sensitive fluorescent probes in a wound infection model, our studies have shown that the portable imager has the ability to detect wound infection in mice. Together, these studies give strong evidence that the portable imager is capable of providing sensitive and quantitative measurements of luminescent and fluorescent signals in real time and in vivo. The results presented here point to a promising future for this device in in vivo imaging applications. The potential of the portable imager for clinical applications will be investigated in the future.

\section{Disclosures}

The authors have nothing to disclose.

\section{Acknowledgments}

Drs. Tang and $\mathrm{Hu}$ report grants from the National Institutes of Health during the work conducted on this study. This work was supported by grants from the National Institutes of Health (GM101776 and AR064650).

\section{References}

1. X. Zhou et al., "A ratiometric fluorescent probe based on a coumarinhemicyanine scaffold for sensitive and selective detection of endogenous peroxynitrite," Biosens. Bioelectron. 64(1), 285-291 (2015).

2. R. A. Bryant and D. P. Nix, Acute \& Chronic Wounds: Current Management Concepts, 4th ed., Elsevier/Mosby, St. Louis, Missouri (2012).

3. S. C. Gnyawali et al., "High-resolution harmonics ultrasound imaging for non-invasive characterization of wound healing in a pre-clinical swine model," PLoS One 10(3), e0122327 (2015).

4. S. Lange-Asschenfeldt et al., "Applicability of confocal laser scanning microscopy for evaluation and monitoring of cutaneous wound healing," J. Biomed. Opt. 17(7), 076016 (2012).

5. C. J. Celeste et al., "Skin temperature during cutaneous wound healing in an equine model of cutaneous fibroproliferative disorder: kinetics and anatomic-site differences," Vet. Surg. 42(2), 147-153 (2013).

6. P. Ghassemi et al., "A polarized multispectral imaging system for quantitative assessment of hypertrophic scars," Biomed. Opt. Express 5(10), 3337-3354 (2014).

7. M. Kuck et al., "Evaluation of optical coherence tomography as a noninvasive diagnostic tool in cutaneous wound healing," Skin Res. Technol. 20(1), 1-7 (2014).

8. A. J. Luber et al., "Characterization of knuckle (Garrod) pads using optical coherence tomography in vivo," Cutis 96(3), E10-E11 (2015).

9. R. Weissleder, "A clearer vision for in vivo imaging," Nat. Biotechnol. 19(4), 316-317 (2001).

10. E. M. Sevick-Muraca, "Translation of near-infrared fluorescence imaging technologies: emerging clinical applications," Annu. Rev. Med. 63(1), 217-231 (2012).

11. V. Ntziachristos, C. Bremer, and R. Weissleder, "Fluorescence imaging with near-infrared light: new technological advances that enable in vivo molecular imaging," Eur. Radiol. 13(1), 195-208 (2003).

12. M. S. Weingarten et al., "Diffuse near-infrared spectroscopy prediction of healing in diabetic foot ulcers: a human study and cost analysis," Wound Repair Regen. 20(6), 911-917 (2012).

13. J. Lei et al., "Quantitative wound healing studies using a portable, lowcost, hand-held near-infrared optical scanner: preliminary sensitivity and specificity analysis," Proc. SPIE 9699, 96990S (2016).

14. T. R. Dargaville et al., "Sensors and imaging for wound healing: a review," Biosens. Bioelectron. 41, 30-42 (2013).

15. J. Zhou et al., "Real-time detection of implant-associated neutrophil responses using a formyl peptide receptor-targeting NIR nanoprobe," Int. J. Nanomed. 7(1), 2057-2068 (2012).

16. J. Zhou et al., "Real time monitoring of biomaterial-mediated inflammatory responses via macrophage-targeting NIR nanoprobes," Biomaterials 32(35), 9383-9390 (2011).

17. E. N. Tang et al., "In vivo imaging of infection using a bacteria-targeting optical nanoprobe,” J. Biomed. Nanotechnol. 10(5), 856-863 (2014).

18. S. Jawhara and S. Mordon, "In vivo imaging of bioluminescent Escherichia coli in a cutaneous wound infection model for evaluation of an antibiotic therapy," Antimicrob. Agents Chemother. 48(9), 34363441 (2004).

19. J. Zhou et al., "Noninvasive assessment of localized inflammatory responses," Free Radical Biol. Med. 52(1), 218-226 (2012).

20. S. Schreml et al., "2D luminescence imaging of $\mathrm{pH}$ in vivo," Proc. Nat. Acad. Sci. U. S. A. 108(6), 2432-2437 (2011). 
21. A. H. Zhou, "A survey of optical imaging techniques for assessing wound healing," Int. J. Intell. Control Syst. 17(3), 79-85 (2012).

22. M. S. Weingarten et al., "Measurement of optical properties to quantify healing of chronic diabetic wounds," Wound Repair Regen. 14(3), 364-370 (2006).

23. E. S. Papazoglou et al., "Near infrared diffuse optical tomography: improving the quality of care in chronic wounds of patients with diabetes," Biomed. Instrum. Technol. 41(1), 83-87 (2007).

24. N. Valim et al., "Experimental measurement of time-dependent photon scatter for diffuse optical tomography," J. Biomed. Opt. 15(6), 065006 (2010).

25. E. S. Papazoglou et al., "Image analysis of chronic wounds for determining the surface area," Wound Repair Regen. 18(4), 349-358 (2010).

26. R. J. Meier et al., "Simultaneous photographing of oxygen and $\mathrm{pH}$ in vivo using sensor films," Angew. Chem. Int. Ed. Engl. 50(46), 1089310896 (2011).

27. R. D. Galiano et al., "Quantitative and reproducible murine model of excisional wound healing," Wound Repair Regen. 12(4), 485-492 (2004).

28. C. Dunnill et al., "Reactive oxygen species (ROS) and wound healing: the functional role of ROS and emerging ROS-modulating technologies for augmentation of the healing process," Int. Wound. J. (2015).

29. W. C. Parks, C. L. Wilson, and Y. S. Lopez-Boado, "Matrix metalloproteinases as modulators of inflammation and innate immunity," Nat. Rev. Immunol. 4(8), 617-629 (2004).
30. O. Saito and H. Furuhata, "Improving uniformity of intensity distribution of ultrasound passing through a human-skull fragment by random modulation," Int. J. Clin. Neurosci. Mental Health 1(Suppl. 1), S23 (2014).

31. S. Roy et al., "Dermal wound healing is subject to redox control," Mol. Ther. 13(1), 211-220 (2006).

32. A. Tanaka, Y. Yamane, and H. Matsuda, "Mast cell MMP-9 production enhanced by bacterial lipopolysaccharide," J. Vet. Med. Sci. 63(7), 811-813 (2001).

33. S. J. Galli, M. Maurer, and C. S. Lantz, "Mast cells as sentinels of innate immunity," Curr. Opin. Immunol. 11(1), 53-59 (1999).

34. B. Xie, Z. Dong, and I. J. Fidler, "Regulatory mechanisms for the expression of type IV collagenases/gelatinases in murine macrophages," J. Immunol. 152(7), 3637-3644 (1994).

35. N. Bryan et al., "Reactive oxygen species (ROS): a family of fate deciding molecules pivotal in constructive inflammation and wound healing," Eur. Cell Mater. 24(1), 249-265 (2012).

36. S. Schreml et al., "Oxygen in acute and chronic wound healing," Br. J. Dermatol. 163(2), 257-268 (2010).

Biographies for the authors are not available. 